\title{
Threshold-crossing statistics in diffusion with a time-dependent control parameter
}

\author{
V Balakrishnan* \\ Department of Physics, Indian Institute of Technology-Madras, Chennai 600 036, India \\ E-mail: voalkiqdysics.iitm.ac.in \\ C. Nicolis \\ Institut Royale Météorologique de Belgique, Avenue Circulaire 3, 1180 Bruxelles, Belgium \\ E-mail: svnGreteo.ara.be

\section{G. Nicolis} \\ Centre for Nonlinear Phenomena and Complex Systems, Université Libre de Bruxelles, Code \\ Postal 231, Boulevard du Triomphe, 1050 Brussels, Belgium \\ E-mail: gnicolis@ullb.ac.be
}

\begin{abstract}
We study two important aspects of the diffusion of a free particle in the presence of a timedependent control parameter. The latter is represented by a friction coefficient that is a given function of time. We solve the stochastic Liouville equation (the Fokker-Planck equation) for the probability density of the particle in phase space, i. e., in both position and velocity. The exact solution is then used to analyze the behavior of (i) the variance in the position, a global characterizer of the system; and (ii) the mean rate of crossings of an arbitrary threshold in the position, a local characterizer. The former is the more conventional descriptor of diffusive processes, but the latter provides valuable complementary information on the dynamical behavior. Depending on the long-time behavior of the friction coefficient, the asymptotic behaviors of both these characterizers vary, and exhibit several cross-overs. This helps elucidate the nature of the interplay between the destabilizing effects of the noise and the stabilizing tendency of the damping, as the latter undergoes a controlled variation in time.
\end{abstract}

International Conference on Statistical Mechanics of Plasticity and Related Instabilities August 29-September 2, 2005

Indian Institute of Science, Bangalore, India

*Speaker. 


\section{Introduction}

The stability of stochastically driven systems is a fundamental problem in dynamics that is also very important from the point of view of applications. Among two general approaches to this question, the more familiar one, via the moments of the dynamical variable(s), enables us to quantify $[1,2]$ the extent of the dispersion about the mean value, the deviation of the mean value from the typical value, the deviation of the distribution from the Gaussian form, and so on. It is evident that the moments represent a global characterization of the system. At a more local level, useful information may be obtained by analyzing the statistics of the crossings of some prescribed threshold value by the stochastic trajectory [3,4], as well as related aspects such as order statistics and extreme value statistics [5]. These two approaches are complementary, and taken together they provide a comprehensive view of the behavior of the system of interest.

In most analyses, it is generally assumed for convenience and tractability that the parameters of the system are time-independent. While this is a reasonable assumption or approximation in many situations, there are numerous other important instances in which it is not valid, ranging from chemical and biological processes to atmospheric phenomena [6] and economics [7]. Time dependence of one or more parameters frequently arises due to a systematic variation caused by external factors, or from externally controlled forcing. Some work has been done on moment dynamics in systems driven by white noise in the presence of time-dependent control parameters $[8,9,10]$. More recently, both moment evolution and threshold crossings have been studied [11] in systems driven by discrete (in particular, dichotomous) noise, the time-varying control parameter being the rate of switching between the levels of the noise.

In view of the ubiquity of systems driven by noise whose correlation time may justifiably be neglected in comparison with the other characteristic time scales present, we revisit some of the simplest models of stochastic flows driven by white noise, but with a time-dependent control parameter. In particular, we shall be interested in the interplay between the destabilizing effects of the noise which leads to unbounded motion in the mean and/or variance, and the stabilizing effect of an appropriate damping coefficient. The time-dependence could be in this coefficient or in the amplitude of the noise (or both), but for definiteness we focus in this paper on the first possibility. Threshold crossings, i. e., the crossings of some prescribed value or 'level' by the system's trajectory, will be one of our primary concerns. Now, it is known that the application of various threshold-crossing formulas to variables directly driven by white noise leads to divergences that arise because of the formally infinite variance of white noise. We circumvent this problem by considering the corresponding flow in phase space. From a technical point of view, this amounts to a tempering of the noise by integration, analogous to the manner in which integrating a Gaussian white noise leads to a better-behaved object, namely, the Wiener process.

The outline of the rest of this paper is as follows. In Sec. 2 we consider the diffusion of a free particle within the framework of the Langevin equation, but in phase space, and with a timedependent friction coefficient $\gamma(t)$. The corresponding Fokker-Planck equation can be solved to yield the time-dependent joint probability density $P(x, v, t)$ in the position and the velocity of the particle. In Sec. 3, we apply the formula for the mean number of threshold crossings to the case at hand, and obtain an exact expression for the mean rate of crossings of an arbitrary threshold by the position $x(t)$ of the particle. The conditions under which this rate becomes independent of the 
amplitude of the noise are identified, and a general formula is obtained for the rate as a functional of $\gamma(t)$. In Sec. 4, using a representative family of functional forms for $\gamma(t)$, we demonstrate systematically how the long-time behavior of the variance undergoes a sequence of cross-overs from super-ballistic $\left(\sim t^{3}\right)$ to standard diffusive $(\sim t)$ behavior, as the time dependence of the friction coefficient is changed from one that decays to zero as $t \rightarrow \infty$, to one that becomes unbounded in this limit. Concomitantly, the asymptotic (long-time) behavior of the mean level-crossing rate exhibits a sequence of cross-overs from the $\sim t^{-1}$ behavior of the frictionless case to a limiting $\sim t^{-1 / 2}$ dependence when the friction coefficient diverges as $t \rightarrow \infty$.

\section{Phase space distribution for free diffusion with time-dependent friction}

The diffusion of a free particle in one dimension is described by the Langevin equation in phase space,

$$
\dot{\mathbf{x}}+\Gamma \mathbf{x}=\zeta(t) .
$$

Here $\mathbf{x}$ is the two-component vector $\left(\begin{array}{ll}x & v\end{array}\right)^{\mathrm{T}}$ where the superscript $\mathrm{T}$ denotes the transpose, $v=$ $\dot{x}, \zeta(t)=\left(\begin{array}{ll}0 & \eta(t)\end{array}\right)^{\mathrm{T}}$, and $\eta(t)$ is a stationary, Gaussian, $\delta$-correlated Markov process (a white noise), with

$$
\langle\eta(t)\rangle=0,\left\langle\eta(t) \eta\left(t^{\prime}\right)\right\rangle=q^{2} \delta\left(t-t^{\prime}\right) .
$$

As stated earlier, we consider a time-dependent friction coefficient, so that the drift matrix $\Gamma$ is given by

$$
\Gamma(t)=\left(\begin{array}{cc}
0 & -1 \\
0 & \gamma(t)
\end{array}\right) .
$$

The corresponding density in phase space, $P(x, v, t)$, obeys a Fokker-Planck-like equation with drift matrix $\Gamma$ and diffusion matrix $D=\operatorname{diag}\left(0, \frac{1}{2} q^{2}\right)\left(q^{2}\right.$ could also be time-dependent, in general). This is the "stochastic Liouville equation"[12]

$$
\frac{\partial P}{\partial t}+v \frac{\partial P}{\partial x}=\gamma(t) \frac{\partial(v P)}{\partial v}+q^{2} \frac{\partial^{2} P}{\partial v^{2}} .
$$

In the usual case, when the friction coefficient $\gamma$ is independent of time, $v(t)$ is of course the Ornstein-Uhlenbeck process. In the present instance, $v(t)$ is no longer a stationary random process.

To solve the master equation for $P$, one defines the matrix Green function

$$
G(t)=\mathscr{T}\left\{\exp \left(-\int_{0}^{t} \Gamma\left(t^{\prime}\right) d t^{\prime}\right)\right\}
$$

where $\mathscr{T}$ denotes the time-ordered product, and the covariance matrix

$$
\sigma(t)=2 \int_{0}^{t} G\left(t^{\prime}\right) D G^{\mathrm{T}}\left(t^{\prime}\right) d t^{\prime}
$$

Then, for sharp initial conditions $\left(x_{0}, v_{0}\right)$, we have $\langle\mathbf{x}(t)\rangle=G(t) \mathbf{x}_{0}$, so that the mean values $\langle x(t)\rangle$ and $\langle v(t)\rangle$ are linear combinations of $x_{0}$ and $v_{0}$ with time-dependent coefficients. The formal solution [13] of the Fokker-Planck equation in this case is a Gaussian in $\mathbf{x}$ of the form

$$
P(x, v, t)=\frac{1}{2 \pi \sqrt{\Delta}} \exp \left\{-\frac{1}{2} S_{11}(\delta x)^{2}-S_{12}(\delta x)(\delta v)-\frac{1}{2} S_{22}(\delta v)^{2}\right\},
$$


where $\delta \mathbf{x}=\mathbf{x}-\langle\mathbf{x}(t)\rangle$ and

$$
\Delta(t)=\operatorname{det} \sigma(t), \quad S(t)=\sigma^{-1}(t) .
$$

As mentioned in the Introduction, we focus here on the case in which the friction coefficient $\gamma(t)$ serves as the time-dependent control parameter, the variance $q^{2}$ of the noise remaining constant in time. We note that the product of two drift matrices of the form specified in Eq. (2.3) satisfies the equation

$$
\Gamma\left(t_{1}\right) \Gamma\left(t_{2}\right)=\Gamma\left(t_{1}\right) \gamma\left(t_{2}\right) .
$$

Owing to this convenient property of the drift matrix, it turns out that $G(t)$ and $\sigma(t)$ can be evaluated in closed form, and the solution for $P(x, v, t)$ written down explicitly. In terms of the functions

$$
\phi(t)=\exp \left(-\int_{0}^{t} \gamma\left(t^{\prime}\right) d t^{\prime}\right), \psi(t)=\int_{0}^{t} \phi\left(t^{\prime}\right) d t^{\prime},
$$

we find that the first moments are given by

$$
\langle x(t)\rangle=x_{0}+v_{0} \psi(t),\langle v(t)\rangle=v_{0} \phi(t) .
$$

Defining the functions

$$
A(t)=\int_{0}^{t} \phi^{2}\left(t^{\prime}\right) d t^{\prime}, \quad B(t)=\frac{1}{2} \psi^{2}(t), \quad C(t)=\int_{0}^{t} \psi^{2}\left(t^{\prime}\right) d t^{\prime},
$$

we get

$$
\sigma(t)=q^{2}\left(\begin{array}{ll}
C & B \\
B & A
\end{array}\right), \quad S(t)=\sigma^{-1}(t)=\frac{q^{2}}{\Delta}\left(\begin{array}{ll}
A & B \\
B & C
\end{array}\right),
$$

where

$$
\Delta(t)=q^{4}\left(A C-B^{2}\right)=q^{4}\left\{\int_{0}^{t} \phi^{2}\left(t^{\prime}\right) d t^{\prime} \int_{0}^{t} \psi^{2}\left(t^{\prime}\right) d t^{\prime}-\frac{1}{4} \psi^{4}(t)\right\} .
$$

For our present purposes (the study of the fluctuations and threshold crossings of the random variable $x(t)$ ), it is helpful to simplify matters by eliminating the systematic drift that arises if $\langle v(t)\rangle$ is non-vanishing. Accordingly, we set the initial velocity $v_{0}=0$, so that $\langle x(t)\rangle=x_{0},\langle v(t)\rangle=0$. The explicit expressions that are then obtained also help us understand more closely, among other things, the dependence of the level-crossing rate on the noise amplitude $q^{2}$. The joint probability density reduces in this drift-free case to

$$
P(x, v, t)=\frac{1}{2 \pi q^{2} \sqrt{A C-B^{2}}} \exp \left\{-\frac{A\left(x-x_{0}\right)^{2}-2 B\left(x-x_{0}\right) v+C v^{2}}{2 q^{2}\left(A C-B^{2}\right)}\right\},
$$

where $A(t), B(t)$ and $C(t)$ have been defined in Eq. (2.12). Integrating Eq. (2.15) over $v$, we obtain the positional probability density

$$
P(x, t)=\frac{1}{\sqrt{2 \pi q^{2} C(t)}} \exp \left\{-\frac{\left(x-x_{0}\right)^{2}}{2 q^{2} C(t)}\right\} .
$$

The fluctuations in $x$ are then completely characterized by the behavior of its variance, which is given by

$$
\sigma_{x}^{2}(t)=q^{2} C(t)
$$

The asymptotic behavior of $C(t)$ is thus an immediate quantitative measure of the degree of stability or instability of the system in various cases. We shall analyze this behavior in some detail in the sequel, after deriving the results required for the analysis of threshold crossings in $x$. 


\section{Threshold-crossing formulas}

We recapitulate briefly some elementary results pertaining to threshold-crossing statistics for a stochastic process $x(t)$ whose sample paths are sufficiently regular-more precisely, mean square differentiable $[3,4]$. Let $x_{\text {th }}$ denote any prescribed threshold value of $x$. Consider the random process $y=\theta\left(x-x_{\text {th }}\right)$, where $\theta(x)$ stands for the unit step function. If crossings of the threshold occur at $t_{i}, i=1,2, \ldots$, we have

$$
\dot{y}=\frac{d}{d t} \theta\left(x-x_{\mathrm{th}}\right)=\sum_{i} \frac{\delta\left(t-t_{i}\right)}{\left|\dot{x}\left(t_{i}\right)\right|} \dot{x}\left(t_{i}\right)
$$

It is at once evident that $\dot{y}$ is a pulse process in time, comprising $\delta$-functions of strength +1 [respectively, -1] at up-crossings [respectively, down-crossings] of $x_{\text {th }}$ by the stochastic trajectory. Hence the number of crossings of the specified level $x=x_{\text {th }}$ in a time interval $\left(t_{1}, t_{2}\right)$ is $N\left(x_{\mathrm{th}} ; t_{1}, t_{2}\right)=\int_{t_{1}}^{t_{2}}|\dot{y}| d t$. The mean value of this number is given in terms of the phase space density $P(x, v, t)$ by

$$
\left\langle N\left(x_{\mathrm{th}} ; t_{1}, t_{2}\right)\right\rangle=\int_{t_{1}}^{t_{2}} d t \int_{-\infty}^{\infty} d v|v| P\left(x_{\mathrm{th}}, v, t\right) .
$$

Both up-crossings and down-crossings of $x_{\text {th }}$ are included in this formula. (The integral over $v$ from $-\infty$ to 0 counts the mean number of down-crossings, while that from 0 to $\infty$ counts the mean number of up-crossings.) The quantity

$$
r\left(x_{\mathrm{th}}, t\right)=\int_{-\infty}^{\infty} d v|v| P\left(x_{\mathrm{th}}, v, t\right)
$$

can be regarded as the instantaneous mean rate of crossings of the threshold concerned.

In the special case when $x(t)$ is a differentiable stationary Gaussian process with zero mean, Eq. (3.3) yields a stationary mean threshold-crossing rate[14]

$$
r^{\mathrm{st}}\left(x_{\mathrm{th}}\right)=\left(\frac{\sigma_{v}}{\pi \sigma_{x}}\right) \exp \left(-\frac{x_{\mathrm{th}}^{2}}{2 \sigma_{x}^{2}}\right) .
$$

As an illustration, we can apply this formula right away to the case of a "Brownian oscillator", i. e., a harmonically bound particle with a constant friction coefficient $\gamma_{0}$, subjected to a Gaussian white noise. The drift matrix in this case is

$$
\Gamma=\left(\begin{array}{cc}
0 & -1 \\
\omega_{0}^{2} & \gamma_{0}
\end{array}\right)
$$

There is, of course, no long-range diffusion in this case. Solving the corresponding Fokker-Planck equation and proceeding to the long-time limit, we obtain

$$
r^{\mathrm{st}}\left(x_{\mathrm{th}}\right)=\left(\frac{\omega_{0}}{\pi}\right) \exp \left(-\frac{\gamma_{0} \omega_{0}^{2} x_{\mathrm{th}}^{2}}{q^{2}}\right)
$$

When $x_{\text {th }}=0$, the dependence on the damping coefficient $\gamma_{0}$ and the noise amplitude $q^{2}$ disappears, and the rate reduces to $\omega_{0} / \pi$, the rate of zero crossings in the undamped, unperturbed oscillatori. e., twice in each period. 
The formulas above are no longer valid if the sample paths of $x(t)$ are not differentiable. This is what happens when $x(t)$ is a one-dimensional diffusion process given by the stochastic differential equation $\dot{x}=f(x, t)+g(x, t) \eta(t)$. The divergent variance of $\dot{x}$ in this case implies that the mean number of crossings of any threshold $x_{\text {th }}$ is infinite in any time interval. On the other hand, if we consider dynamics in phase space, and it is the velocity $v$ rather than $x$ itself that is driven by the noise $\eta(t)$, then the process $x(t)$ is sufficiently smooth to permit meaningful threshold-crossing statistics. It is this fact that we exploit in order to obtain threshold-crossing statistics for freeparticle diffusion in the presence of an explicitly time-dependent friction coefficient.

We substitute Eq. (2.15) for $P(x, v, t)$ in Eq. (3.3) and evaluate the integrals concerned. After simplification, we finally arrive at the result

$$
\begin{aligned}
r\left(x_{\mathrm{th}}, t\right)= & \frac{\sqrt{A C-B^{2}}}{\pi C} \exp \left(-\frac{A\left(x_{\mathrm{th}}-x_{0}\right)^{2}}{2 q^{2}\left(A C-B^{2}\right)}\right) \\
& +\frac{B\left(x_{\mathrm{th}}-x_{0}\right)}{C \sqrt{2 \pi C q^{2}}} \exp \left(-\frac{\left(x_{\mathrm{th}}-x_{0}\right)^{2}}{2 C q^{2}}\right) \operatorname{erf}\left(\frac{B\left(x_{\mathrm{th}}-x_{0}\right)}{\sqrt{2 C q^{2}\left(A C-B^{2}\right)}}\right),
\end{aligned}
$$

where the functions $A(t), B(t)$ and $C(t)$ have been defined in Eq. (2.12). As $q^{2} \rightarrow 0$, the thresholdcrossing rate vanishes extremely rapidly, like $\exp \left(-1 / q^{2}\right)$. On the other hand, as $q^{2} \rightarrow \infty$, it saturates to a value $\sqrt{A C-B^{2}} /(\pi C)$. The same expression is obtained, for arbitrary $q^{2}$, for the the rate of crossings of the starting point $x_{0}$ itself: all dependence on the noise amplitude disappears in this case, and we get

$$
r\left(x_{0}, t\right)=\left(\int_{0}^{t} \phi^{2}\left(t^{\prime}\right) d t^{\prime} \int_{0}^{t} \psi^{2}\left(t^{\prime}\right) d t^{\prime}-\frac{1}{4} \psi^{4}(t)\right)^{1 / 2} /\left(\pi \int_{0}^{t} \psi^{2}\left(t^{\prime}\right) d t^{\prime}\right) .
$$

Equation (3.8) provides us with a convenient and compact formula amenable to further analysis.

\section{Behavior of the variance and the mean threshold-crossing rate}

We are now ready to study the behavior of the variance of $x$, as given by Eq. (2.17), and the mean threshold-crossing rate, as given by Eq. (3.8), in detail.

As our main interest is in the long-time behavior of these quantities, let us first dispose of the short-time $(t \rightarrow 0)$ behavior very briefly. Since $\phi(t) \simeq 1, \psi(t) \simeq t$ in this limit, the leading shorttime behavior of $\sigma_{x}^{2}(t)$ and $r\left(x_{0}, t\right)$ is universal in the sense of being completely noise-dominated and independent of $\gamma(t)$ : We find the super-ballistic behavior $\sigma_{x}^{2}(t) \simeq q^{2} t^{3} / 3$, as expected, while $r\left(x_{0}, t\right) \simeq \sqrt{3} /(2 \pi t)$. As we have already pointed out, the divergence of the threshold-crossing rate precisely at $t=0$ is unavoidable in white-noise-driven systems. For sufficiently short times, it implies a logarithmic growth of the mean number of crossings up to time $t$ starting from an initial time $t_{0}>0$.

As $t$ increases, however, the time-dependence of these quantities becomes more intricate. The long-time or asymptotic behavior, in particular, is strongly dependent on that of $\gamma(t)$. In order to exhibit systematically how significant changes in threshold-crossing statistics can be induced by suitable time variations of the control parameter $\gamma(t)$, we model the latter by a representative family of functions, given by

$$
\gamma(t)=\gamma_{0}\left(\frac{\tau}{t+\tau}\right)^{\alpha}
$$


Here $\gamma_{0}$ represents a scale for the strength of the friction coefficient, $\tau$ provides a time scale, while the real exponent $\alpha$ serves to characterize both decreasing and increasing functions $\gamma(t)$, depending on whether $\alpha>0$ or $\alpha<0$. We shall follow a progression of values of $\alpha$ (and the other parameters, $\gamma_{0}$ and $\tau$ ) representing, in the long-time limit, a range from small friction to large friction. This will serve to bring out clearly the gradual cross-overs in the leading asymptotic behavior of the variance and the mean crossing rate. The set of functions in Eq. (4.1) essentially comprises only power laws in $t$, but this suffices to make our point: even small changes in the exponent $\alpha$ can have a significant effect on the asymptotic behavior of the system. It turns out that the value $\alpha=1$ separates qualitatively different kinds of asymptotic behavior, because the latter depends crucially on whether the function $\phi(t)$ defined in Eq. (2.10) vanishes or tends to a nonzero limit as $t \rightarrow \infty$. Exactly at the borderline value $\alpha=1$, the behavior further depends on the value of $\gamma_{0} \tau$. We omit the details and present only the results for the dominant large- $t$ dependence of $\sigma_{x}^{2}(t)$ and $r\left(x_{0}, t\right)$.

(i) $\alpha>1$ : In this case $\phi(\infty)=\exp \left[-\gamma_{0} \tau /(\alpha-1)\right]$ is a positive number $(<1)$, so that $\psi(t) \rightarrow t \phi(\infty)$ for large $t$. The decay of the friction coefficient is rapid enough to ensure that the asymptotic longtime behavior of $\sigma_{x}^{2}(t)$ and $r\left(x_{0}, t\right)$ is again similar to that in the complete absence of friction. We find

$$
\sigma_{x}^{2}(t) \rightarrow \frac{1}{3} q^{2} \phi^{2}(\infty) t^{3}, \quad r\left(x_{0}, t\right) \rightarrow \frac{\sqrt{3}}{2 \pi t} .
$$

At intermediate times, of course, $r\left(x_{0}, t\right)$ deviates from the exact result $\sqrt{3} /(2 \pi t)$ of the zerofriction case: the first correction at short times is $\sqrt{3} \gamma_{0} /(8 \pi)$, which happens to be independent of the value of $\alpha$.

When $\alpha=1, \phi(t)$ decays to zero according to a power law. This leads to a sequence of subcases, as follows.

(ii) $\alpha=1, \gamma_{0} \tau<\frac{1}{2}$ : The leading behavior of $\sigma_{x}^{2}$ is given by

$$
\sigma_{x}^{2}(t) \rightarrow \frac{q^{2} \tau^{2 \gamma_{0} \tau} t^{3-2 \gamma_{0} \tau}}{\left(1-\gamma_{0} \tau\right)^{2}\left(3-2 \gamma_{0} \tau\right)}
$$

The behavior of the variance is still super-ballistic, with an exponent lying between 2 and 3 . The threshold-crossing rate has a leading behavior

$$
r\left(x_{0}, t\right) \rightarrow \frac{1}{2 \pi t}\left(\frac{3-2 \gamma_{0} \tau}{1-2 \gamma_{0} \tau}\right)^{1 / 2},
$$

showing how the coefficient of $(2 \pi t)^{-1}$ starts building up as the friction begins to enter the picture.

(iii) $\alpha=1, \gamma_{0} \tau=\frac{1}{2}$ : The foregoing coefficient now diverges. Equation (4.3) shows that $\sigma_{x}^{2}(t) \sim t^{2}$ in this case, corresponding to ballistic motion. We also find

$$
r\left(x_{0}, t\right) \rightarrow \frac{1}{\sqrt{2} \pi t} \ln ^{1 / 2}\left(\frac{t}{\tau}\right)
$$

signaling the start of a deviation from the $t^{-1}$ power law, by a $\sqrt{(\ln t)}$ modulating factor. 
(iv) $\alpha=1, \frac{1}{2}<\gamma_{0} \tau<1$ : It follows from Eq. (4.3) that the variance diverges like a power of $t$ lying between 1 and 2 , so that the motion is super-diffusive; while

$$
r\left(x_{0}, t\right) \rightarrow \frac{\left(1-\gamma_{0} \tau\right)}{\pi \tau}\left(\frac{3-2 \gamma_{0} \tau}{2 \gamma_{0} \tau-1}\right)^{1 / 2}\left(\frac{\tau}{t}\right)^{-\gamma_{0} \tau+3 / 2}
$$

Thus the fall-off of the mean threshold-crossing rate has switched to a power law with an exponent that lies in between -1 and $-\frac{1}{2}$. This behavior continues till we reach $\gamma_{0} \tau=1$.

(v) $\alpha=1, \gamma_{0} \tau=1$ : In this case we have an asymptotic behavior of the variance that is marginally super-diffusive in the sense of a logarithmic modulating factor,

$$
\sigma_{x}^{2}(t) \rightarrow q^{2} \tau^{2} t \ln ^{2}\left(\frac{t}{\tau}\right)
$$

Correspondingly,

$$
r\left(x_{0}, t\right) \rightarrow \frac{1}{\pi \ln \left(\gamma_{0} t\right)}\left(\frac{\gamma_{0}}{t}\right)^{1 / 2},
$$

The exponent has become $-\frac{1}{2}$, but with a $(\ln t)^{-1}$ modulation of the power law.

(vi) $\alpha=1, \gamma_{0} \tau>1$ : We find

$$
\sigma_{x}^{2}(t) \rightarrow \frac{q^{2} \tau^{2} t}{\left(\gamma_{0} \tau-1\right)^{2}}
$$

while

$$
r\left(x_{0}, t\right) \rightarrow \frac{\left(\gamma_{0} \tau-1\right)}{\pi\left[\left(2 \gamma_{0} \tau-1\right) \tau t\right]^{1 / 2}} .
$$

The variance has thus attained its standard asymptotic diffusive behavior, while the cross-over of $r\left(x_{0}, t\right)$ to a $t^{-1 / 2}$ decay is complete.

(vii) $\alpha<1$ : The function $\phi(t)$ now approaches zero as $t \rightarrow \infty$ at least as rapidly as a stretched exponential. Therefore $\psi(\infty)$ and $A(\infty)$ are finite quantities, enabling us to derive the general asymptotic formulas

$$
\sigma_{x}^{2}(t) \rightarrow q^{2}\left(\int_{0}^{\infty} d t^{\prime} \phi\left(t^{\prime}\right)\right)^{2} t
$$

and

$$
r\left(x_{0}, t\right) \rightarrow \frac{\left(\int_{0}^{\infty} d t^{\prime} \phi^{2}\left(t^{\prime}\right)\right)^{1 / 2}}{\pi\left(\int_{0}^{\infty} d t^{\prime} \phi\left(t^{\prime}\right)\right)} t^{-1 / 2} .
$$

This remains valid for all $\alpha<1$, including negative values of this exponent. At $\alpha=0$, we recover the results

$$
\sigma_{x}^{2}(t) \rightarrow \frac{q^{2} t}{\gamma_{0}^{2}}, \quad r\left(x_{0}, t\right) \rightarrow\left(\frac{\gamma_{0}}{2 \pi^{2}}\right)^{1 / 2} t^{-1 / 2}
$$

corresponding to the case of a constant friction coefficient $\gamma_{0}$. 
(viii) $\alpha=-1$ : This case is of special interest: the friction coefficient increases linearly with time as on a ramp, according to $\gamma(t)=\gamma_{0}+\varepsilon t$ where $\varepsilon \equiv \gamma_{0} / \tau$. Then

$$
\sigma_{x}^{2}(t) \rightarrow \frac{\pi q^{2}}{2 \varepsilon} e^{\gamma_{0}^{2} / \varepsilon}\left[1-\operatorname{erf}\left(\gamma_{0} / \sqrt{2 \varepsilon}\right)\right]^{2} t
$$

and

$$
r\left(x_{0}, t\right) \rightarrow \frac{\varepsilon^{1 / 4}\left[1-\operatorname{erf}\left(\gamma_{0} / \sqrt{\varepsilon}\right)\right]^{1 / 2}}{\pi^{5 / 4}\left[1-\operatorname{erf}\left(\gamma_{0} / \sqrt{2 \varepsilon}\right)\right]} t^{-1 / 2} .
$$

As already mentioned, this asymptotic behavior implies that, for any $t_{0}>0$, the mean number of crossings $\left\langle N\left(x_{0} ; t_{0}, t\right)\right\rangle$ increases like $t^{1 / 2}$ as $t \rightarrow \infty$.

All the results reported above have also been verified by extensive numerical simulation.

\section{Acknowledgments}

VB acknowledges the warm hospitality of the Université Libre de Bruxelles, where this work was initiated. This work was supported in part by the Interuniversity Attraction Poles Program of the Belgian Federal Government and the Belgian National Fund for Scientific Research.

\section{References}

[1] G. Nicolis and I. Prigogine, Self-Organization in Non-Equilibrium Systems (Wiley, New York, 1977).

[2] N. G. Van Kampen, Stochastic Processes in Physics and Chemistry (North-Holland, Amsterdam, 1981).

[3] R. L. Stratonovich, Topics in the Theory of Random Noise, Vol. II (Gordon and Breach, New York, 1967).

[4] T. T. Soong, Random Differential Equations in Science and Engineering (Academic, New York, 1973).

[5] M. R. Leadbetter, G. Lindgren, and H. Rootzen, Extremes and Related Properties of Random Sequences and Processes (Springer-Verlag, New York, 1983).

[6] C. Nicolis, Tellus 40A, 50 (1988).

[7] P. Embrechts, C. Klüppenberg, and T. Mikosch, Modelling Extreme Events for Insurance and Finance (Springer-Verlag, New York, 1997).

[8] T. Erneux and P. Mandel, SIAM J. Appl. Math. 46, 1 (1986).

[9] S. Belestri, M. Ciofini, R. Meucci, F. Arecchi, P. Collet, M. San Miguel, and S. Balle, Phys. Rev. A 44, 5894 (1991).

[10] C. Nicolis and G. Nicolis, Phys. Rev. E 62, 192 (2000).

[11] G. Nicolis, V. Balakrishnan, and C. Nicolis, Phys. Rev. E 65, 051109 (2002).

[12] S. Chandrasekhar, Rev. Mod. Phys. 15, 1 (1943).

[13] H. Risken, The Fokker-Planck Equation (Springer-Verlag, New York, 1984).

[14] S. O. Rice, Bell System Tech. J. 23, 282 (1944); ibid. 24, 46 (1945). 